\title{
Advances in pre-hospital care - operational experiences from the Melbourne mobile stroke unit
}

\author{
Henry Zhao, ${ }^{1,2}$, Bruce C. V. Campbell',2, Shane Foster ${ }^{2}$, Michael Stephenson'2, Skye Coote', Francesca \\ Langenberg', Damien Easton', Geoffrey A. Donnan ${ }^{1, \#,}$, Stephen M. Davis ${ }^{1, \#}$
}

'Departments of Medicine and Neurology, Melbourne Brain Centre, Royal Melbourne Hospital, University of Melbourne, Melbourne 3050, Australia.

${ }^{2}$ Clinical Services, Ambulance Victoria, Melbourne 3108, Australia.

\#Joint senior author.

Correspondence to: Dr. Henry Zhao, Department of Neurology, Royal Melbourne Hospital, University of Melbourne, 300 Grattan St, Victoria, Parkville 3050, Australia.E-mail: zhaohdr@live.com

How to cite this article: Zhao H, Campbell BCV, Foster S, Stephenson M, Coote S, Langenberg F, Easton D, Donnan GA, Davis SM. Advances in pre-hospital care-operational experiences from the Melbourne mobile stroke unit. Vessel Plus 2021;5:10. http://dx.doi.org/10.20517/2574-1209.2020.77

Received: 26 Nov 2020 First Decision: 18 Dec 2020 Revised: 30 Dec 2020 Accepted: 8 Jan 2021 Published: 7 Feb 2021

Academic Editor: Aaron S. Dumont Copy Editor: Xi-Jun Chen Production Editor: Yue-Yue Zhang

\begin{abstract}
Mobile stroke units (MSU) are specially designed ambulances with CT imaging capability able to provide faster pre-hospital care to patients in the community. The Melbourne Mobile Stroke Unit is the first MSU service in the Australian region, commencing operations in 2017 in central metropolitan Melbourne. This review describes the challenges of setting up the MSU in a unique Australian setting and initial clinical efficacy of pre-hospital MSU treatment and triage on ischemic and hemorrhagic stroke. We also discuss the current technological and operational limitations of the MSU service and directions for future research.
\end{abstract}

Keywords: Stroke, treatment, thrombolysis, intracerebral hemorrhage

\section{INTRODUCTION}

Mobile stroke units (MSU) are pioneering services incorporating imaging capability and a specialized stroke team in an ambulance platform in order to provide pre-hospital treatment and triage ${ }^{[1]}$. The Melbourne $\mathrm{MSU}^{[2]}$ launched in November 2017 and is the first operational service in Australia and the Oceania region, following its introduction in Europe ${ }^{[3,4]}$ and the United States ${ }^{[5]}$. In this review we describe operationalization of the Melbourne MSU service, the primary benefits on pre-hospital management of

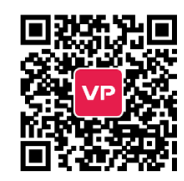




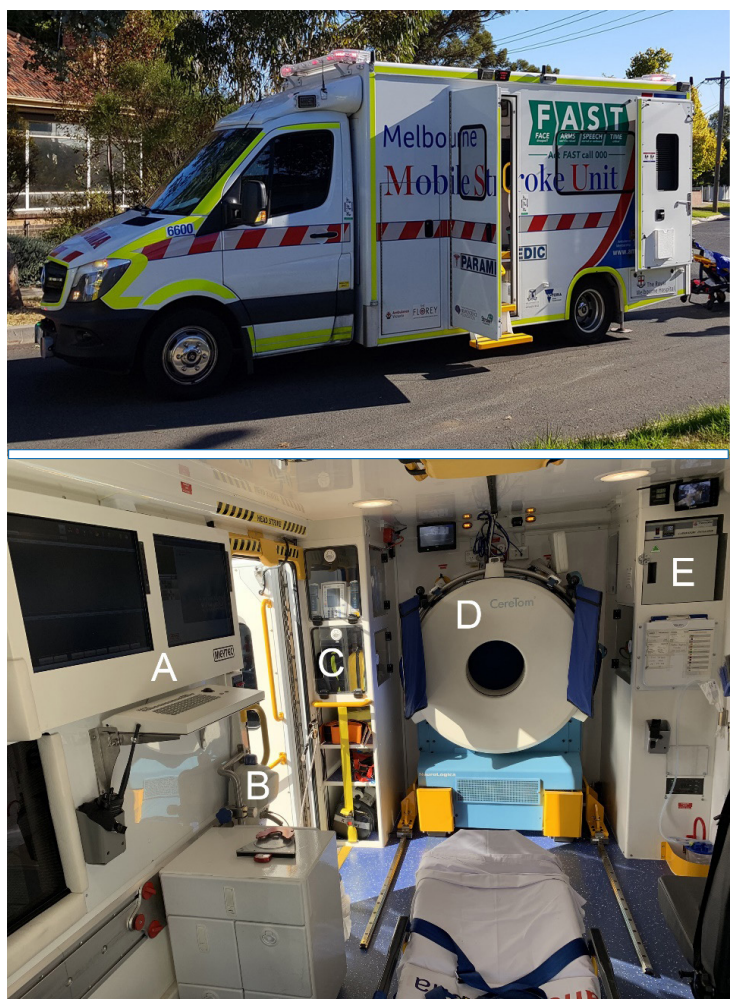

Figure 1. Melbourne mobile stroke unit exterior and interior. mobile stroke unit interior hardware: monitors for viewing and sending imaging in real-time (A); contrast injector (B); storage compartments for medications (C); CereTom scanner (D); contrast warmer (E).

ischemic and intracranial hemorrhage, as well as future research directions and challenges.

\section{Operationalization of the Melbourne mobile stroke unit}

The Melbourne MSU was built with a view to local practices and Australian regulations for emergency vehicles [Figure 1]. The Mercedes Sprinter ${ }^{\mathrm{TM}}$ chassis has previously been successfully fitted for the secondgeneration Homburg $\mathrm{MSU}^{[3]}$ and was chosen for the Melbourne MSU as a smaller vehicle profile to suit local conditions, which consist of areas of high living density and narrow roads sharing traffic with an extensive network of trams. The Melbourne service uses the self-mobile 8-slice CereTom CT scanner (Samsung Neurological Corp, MA), which is the most widely used scanner in MSUs worldwide as they are significantly smaller and lighter than a standard in-hospital CT. The scanner is capable of non-contrast CT brain and contrast-enhanced CT-angiogram of the intracranial circulation (the small bore hole does not permit imaging of the lower neck and thorax). Other hardware features on the Melbourne MSU include $4 \mathrm{G}$ mobile transmission capabilities, in-vehicle audio-visual telemedicine connection, contrast injector, contrast warmer, fridge, and portable point-of-care international normalized ratio testing.

Although previous international MSU services had already been in operation for some years prior to the Melbourne MSU, there were unique challenges to the Australian setting. One example was that the emergency stop button of the CereTom scanner was located on the right side of the machine (if looking from the front of the machine). This usually aids accessibility of the button from the side door in righthand side driving countries, as the side door would be located on the correct side to avoid opening into traffic. Therefore, operators in other countries can physically stand outside the vehicle during a scan, as the emergency stop button could be easily pressed if necessary. Given the left-hand side driving regulations in Australia, the radiographer instead needed to be inside the vehicle and wear lead gowning for radiation protection. 
Further challenges were encountered in the strict no lift policies employed by the local ambulance service (Ambulance Victoria), where all stretchers are hoisted into the ambulance using an electronic floormounted loader. This is opposed to the more simplistic manual lifting mechanisms for stretcher hoisting used in some international services. Under normal circumstances the loader mechanism does not allow the stretcher to be lifted or lowered whilst inside the ambulance. However, due to the need for patient positioning to align with the CT scanner, a technical workaround was required to manually disengage the stretcher from the loader whilst inside the MSU. This procedure makes the patient loading workflow more complex and requires two trained paramedics to operate the stretcher loading sequence.

The staffing composition of the Melbourne MSU, with a neurologist/senior stroke fellow, nurse specialist, radiographer, and two paramedics (one with airway and cardiac resuscitation training), was chosen to reflect a standard in-hospital acute stroke team in Australia. The addition of a paramedic with advanced level training was to cater for stroke patients with unstable conscious state or at risk of neurological deterioration. In comparison to international services, this is a large and highly experienced team, especially as some units operate without neurologists or do not have stroke nurse specialists. Many also utilize CT or emergency technicians who generally have less training than their Australian counterparts. This level of experience has undoubtedly enabled optimized workflows on the Melbourne MSU, compared to a typically more junior in-hospital team.

General operational workflows for the Melbourne MSU have been previously described ${ }^{[2]}$. The operating hours (weekdays from $8 \mathrm{AM}-6 \mathrm{PM}$ ) was decided in part from modelling and by logistical considerations for staff. The operational hours were designed to capture approximately $64 \%$ of all suspected stroke dispatches within the 20-kilometre primary co-dispatch radius, whilst simultaneously being able to be broken into two $5 \mathrm{~h}$ morning and afternoon shifts. The estimated workload appears to be consistent with the experience of the first $24 \mathrm{~h} \mathrm{MSU}$ based in Toledo, USA where the vast majority of dispatches occurred from 7AM-7PM and the bulk of the thrombolysis cases occurred between approximately $10 \mathrm{AM}-6 \mathrm{PM}^{[6]}$.

The primary dispatch radius of the Melbourne MSU was set at 20-kilometres of the home base of Royal Melbourne Hospital. Within this zone, the MSU transports patients to 9 metropolitan stroke centers, of which 4 have 24 h capability for endovascular thrombectomy and neurosurgery. This radius was based on the estimated average on-scene time of $28 \mathrm{~min}$ for Ambulance Victoria attending stroke patients in metropolitan Melbourne (unpublished data, Ambulance Victoria, 2017). As the MSU uses a co-dispatch model, a closer local ambulance crew would arrive and commence extrication with the expectation that the MSU would arrive before they were ready to transport to hospital within this timeframe. Compared to international services, both the Berlin and Houston metropolitan MSUs use a dispatch radius that approximates a $15 \mathrm{~min}$ drive $e^{[4,5]}$ or 7 miles $^{[7]}$ (11.3 kilometres) due to the faster on-scene times of their local ambulance services.

Following a period of operation, the Melbourne MSU began accepting suspected stroke cases outside the 20-kilometre central Melbourne radius where initial attending paramedics had requested the service. In many of these cases the nearest stroke center was in the direction of the MSU base and there was an opportunity for the service to rendezvous with the initial ambulance prior to arrival at hospital. This approach has been successfully by the Houston MSU to double their standard response radius from the usual 6 miles to 12 miles $^{[7]}$. Whilst attending these cases may prevent some concurrent dispatches within the usual 20-kilometre radius, these paramedic-screened cases have a higher specificity for stroke and therefore a higher likelihood of benefitting from MSU management. Should Melbourne paramedics also identify the patient has having a severe stroke syndrome using the ACT-FAST triage algorithm ${ }^{[8]}$, there is a good chance that the patient may require bypass of the peripherally based hospital for an endovascular thrombectomy (EVT) center that is usually based in central Melbourne. 


\section{Benefits for thrombolysis}

The most prominent feature of MSUs is the ability to provide faster pre-hospital thrombolysis. After the first 365 days of operation, a total of 100 patients had received pre-hospital thrombolysis. Time from dispatch of first ambulance to commencement of reperfusion therapy was compared between MSU and a similar cohort of patients in metropolitan Melbourne presenting via regular ambulance to hospital during MSU operating hours ${ }^{[2]}$. Overall results show time to treatment was substantially cut by $42.5 \mathrm{~min}$ (95\%CI: 36.0-49.0). Not surprisingly, the major contributor was avoidance of need to transport to hospital prior to thrombolysis (median dispatch time to scene arrival and hospital arrival was $22.5 \mathrm{~min}$ and 49 min, respectively), but the efficient workflows of the MSU upon arrival was also faster than transport to a thrombolysis center (median time from scene arrival and hospital arrival to treatment was $41.5 \mathrm{~min}$ and 57.0 min, respectively). Therefore, in almost all scenarios, the Melbourne MSU would likely provide faster treatment so long as the service could physically reach the patient prior to hospital arrival and there were no clear difficulties in performing CT scanning or provision of thrombolysis.

Compared to international services, the Melbourne MSU has a somewhat longer time than other busy metropolitan units for some metrics, such as dispatch and scene arrival to thrombolysis ${ }^{[2]}$. Part of this may be due to the higher complexity of cases, as around one-third of patients receiving thrombolysis on the Melbourne service also have large vessel occlusion, therefore making extrication slower. The Melbourne MSU also has a more difficult stretcher loading and positioning process to minimize manual lifting, which takes more time than similar international services. Furthermore, comparison may also be confounded if different services have varying thresholds as to selection of attended patients for MSU management (e.g., if only straight-forward patients are selected for scanning). Despite this, the overall time savings for both onset and dispatch to thrombolysis (compared to local controls) were greater for the Melbourne MSU than for other international services. This suggests that significant on-scene difficulties are faced by regular ambulance transport pathways and the extra staffing of the MSU may ultimately assist with extrication processes.

Another key metric of interest was the ability of the Melbourne MSU to provide very early thrombolysis. Experiences from international MSUs have shown substantial increases in the proportion of patients able to receive thrombolysis within the first hour of known onset, or the "golden hour", compared to usual hospital pathways ${ }^{[9,10]}$. The Melbourne MSU was able to provide golden hour thrombolysis for around $15 \%$ of all treated patients, compared to just $1.5 \%$ in the local control group, indicating a ten-fold increase ${ }^{[2]}$. The largest reported cohort from the Berlin MSU initially reported increase in golden hour thrombolysis from $5 \%$ to $31 \%{ }^{[9]}$, but more recent updates show the figure at $13 \%{ }^{[11]}$ and much closer to the Melbourne service. Three US-based MSUs also reported golden hour thrombolysis between $27 \%-33 \%^{[12-14]}$ but all with very small patient numbers $(<20)$. The proportion of non-MSU patients receiving golden hour thrombolysis in-hospital was reported as $1.3 \%$ in a very large US treatment cohort $(n=65,384 \text { patients })^{[15]}$, which is also very close to that of metropolitan Melbourne.

Finally, the Melbourne MSU is also able to administer supportive medications more efficiently. This includes anti-hypertensive agents pre- and post-thrombolysis, or intravenous idarucizumab to reverse dabigatran prior to thrombolysis ${ }^{[16]}$. This is because the medications are always kept in a known location on the vehicle, compared to sourcing the drug in a large emergency department or pharmacy department (and may require pre-approval from another specialty). MSU personnel are also especially trained to administer these medications efficiently, whereas in-hospital such drugs may need to be given by a general emergency nurse with less experience. 


\section{Benefits for endovascular thrombectomy}

In contrast to published literature on the benefits of MSUs on intravenous thrombolysis, little has been comparatively reported on endovascular thrombectomy. Although the procedure is performed in hospital, the role of accurate triage and early notification of neuro-interventional services has been a proposed advantage of MSUs ${ }^{[17]}$. The lack of early data may be in part due to the unique organization of stroke services in the bigger pioneering MSUs, such as in Berlin, Germany where the majority of stroke centers have EVT capability, meaning MSUs are not able to provide a triage service locally ${ }^{[11]}$.

The Melbourne MSU is therefore one of the very first services to report on the efficacy of MSU-enabled triage in reducing time to EVT commencement ${ }^{[2]}$. In the first 365 days of operation, the MSU had facilitated endovascular thrombectomy for 42 patients. Using a comparator of all direct and metropolitan secondary transfer patients to the largest EVT center in Victoria (Royal Melbourne Hospital), patients receiving facilitated thrombectomy via the Melbourne MSU did so a median of $51 \mathrm{~min}$ (95\%CI: 30-72) faster. On further analysis, cases that bypassed of the local non-EVT hospital received the most time saving of 71 min (95\%CI: 46-96), whereas the equivalent time saving if the patient was located closest to a comprehensive center was 6 min (95\%CI: 19-31). This suggests that a large proportion of the time savings are due to improved triage and avoidance of secondary transfers.

Further contributions to faster EVT were for time from hospital arrival to EVT commencement, which was 17 min (95\%CI: 8-26) faster for MSU patients compared to in-hospital controls. This is likely attributed partly to early pre-hospital notification of neuro-interventional services to prepare the angiography suite for an impending case. Another factor was that one-third of MSU patients received EVT without need for repeat in-hospital imaging. Generally, repeat imaging after arrival in hospital with the addition of CTperfusion is required if the onset time is $>6 \mathrm{~h}^{[18,19]}$ or if the MSU CT-angiogram is not adequate to show the relevant occlusion. However, at the start of the Melbourne MSU the conservative approach was to repeat imaging for all potential EVT cases, although this policy was subsequently relaxed as the comprehensive centers became more familiar with MSU. Therefore, the proportion of MSU EVT cases that need repeat imaging will likely decrease with ongoing operation. In comparison, the time savings for hospital arrival to EVT commencement for US-based MSUs were 10 min or less ${ }^{[20,21]}$.

Despite the lack of statistically significant time saving for EVT patients that did not need bypass, the twothirds that were eligible for thrombolysis still received intravenous therapy substantially faster. Around 10\% of all large vessel occlusions managed by the Melbourne MSU did not proceed to the angiography suite due to improving symptoms or recanalisation from thrombolysis. There are almost certainly further cases that proceeded to angiography but did not require intervention due to recanalisation. Analysis of data from two US-based MSUs showed that large vessel occlusion recanalisation solely with thrombolysis was greater than with in-hospital controls $(29.4 \% v \text { s. } 22 \%, P=0.04)^{[22]}$. This suggests that there is still benefit from MSUs attending potential EVT cases even if the patient is located near a comprehensive center, especially if the patient is thrombolysis-eligible. In addition, not all EVT centers in metropolitan Melbourne are able to run multiple angiography laboratories simultaneously, or all laboratories may be engaged in active cases. This means that MSU personnel can confirm angiography and neuro-intervention availability prior to hospital arrival and, if necessary, divert to another EVT center.

\section{Benefit on disability avoidance from reperfusion therapy}

In addition to time savings, we also examined the possible disability avoidance of providing earlier thrombolysis and EVT, given improved post-stroke outcomes are highly associated with faster reperfusion therapy ${ }^{[23]}$. This was based on previous literature that estimated the overall avoided disability-adjusted life days for each minute of faster thrombolysis ${ }^{[24]}$ and EVT (in addition to thrombolysis) ${ }^{[25]}$, as derived from previous trial data. Using these estimates, it was calculated that in the first 365 operational days, the time 
savings from faster thrombolysis on the MSU would have avoided 20.9 disability-adjusted life years, and 24.6 disability-adjusted life years for faster $\mathrm{EVT}^{[2]}$. This suggests that despite the smaller number of cases, the more powerful effects of EVT compared to thrombolysis meant that faster EVT is the more clinically impactful advantage conferred by the MSU.

This is also predicated on a linear assumption of outcome decay with time, which is likely not true as the benefits for reperfusion therapy decrease fastest in the early timepoint ${ }^{[23,26]}$. Given the data on the dramatic improvement in golden hour thrombolysis, the early timepoints are clearly where the Melbourne MSU is having an impact, meaning our disability avoidance estimates may be conservative.

\section{Benefits for hemorrhagic stroke}

Whilst the MSU platform was conceived primarily for treatment of ischemic stroke, triage and pre-hospital treatment also benefits patients with intracranial hemorrhage. Of all hemorrhage patients attended to by the MSU in the first 365 operational days, $28 \%$ received a CT scan within 60 min of symptom onset and $60 \%$ within 90 min. In contrast, $15 \%$ and $47 \%$ of ischemic stroke patients received thrombolysis within 60 and 90 min respectively on the MSU. This suggests that MSUs can intervene in intracranial hemorrhage at a similarly fast time window as ischemic stroke, which is critical as hematoma growth is highly timedependent ${ }^{[27]}$. In addition, our data also show that almost half of all hemorrhage patients (48\%) have proven or suspected hematoma growth (suspected cases are those who clinically deteriorated). This figure is substantially higher than the placebo arms of past hemostatic trials ${ }^{[28-30]}$, likely due to the earlier assessment and imaging enabled by the MSU. MSUs therefore have the unique role in providing ultra-early therapies in intracranial hemorrhage and determining their potential role in functional outcomes.

Although effective treatments are much more limited for parenchymal intracerebral hemorrhage, a posthoc analysis of a major trial of aggressive blood pressure management in intracerebral hemorrhage demonstrated improved outcomes if anti-hypertensive therapy is initiated within $2 \mathrm{~h}$ of symptom onset ${ }^{[3]}$. In the first 365 days, $42 \%$ of MSU hemorrhage patients required acute intravenous anti-hypertensive therapy, which would be delayed if patients received standard ambulance transport. Pre-hospital imaging and management by the MSU therefore allows faster treatment for blood pressure and potentially greater stabilization of hematoma growth.

The other major therapy provided by the MSU is reversal of anti-coagulation associated hemorrhage. The Melbourne MSU provides reversal for patients on therapeutic warfarin using intravenous vitamin $\mathrm{K}$ and 3 -factor prothrombin concentrate ( $6 \%$ of all intracerebral hemorrhage in the first 365 days, $n=3$ ) and also for dabigatran-associated hemorrhage with intravenous idarucizumab. Although specific reversal therapy for rivaroxaban and apixaban exists in the form of andexanet-alpha ${ }^{[32]}$, this is not yet available for use in Australia. The MSU again has substantial advantages in providing earlier treatment, as there is preliminary evidence that delayed warfarin reversal is associated with poorer outcomes ${ }^{[33]}$.

Similar to large vessel occlusion, pre-hospital diagnosis of intracranial hemorrhage by the MSU allowed improved triage of patients directed to a comprehensive center with neurosurgical units. Our data shows around a quarter of all hemorrhage patients bypassed their local hospital, some of which subsequently underwent emergent neurosurgical intervention. This treatment would have been severely delayed had patients needed a secondary inter-hospital transfer, as transfer processes are even slower than for EVT in Melbourne due to the absence of an established protocol. Such delays are likely to be especially deleterious to younger patients with rapidly progressive hemorrhages, where earlier neurosurgical intervention may delay, reduce, or even prevent disability. Even if patients did not receive surgical intervention, high risk cases require management at a neurosurgical center for neuro-critical care monitoring, especially 
subarachnoid and subdural hemorrhage. There is also evidence to suggest that hemorrhage cases have better outcomes at neurosurgical centers, regardless of whether surgery was performed ${ }^{[34]}$.

In addition, almost a third of all patients with parenchymal hemorrhage were recruited into a currently running clinical trial of tranexamic acid administered within $2 \mathrm{~h}$ of onset (ClinicalTrials.gov Identifier: NCT03385928). Previous hemostatic trials ${ }^{[28-30]}$ in intracerebral hemorrhage have only demonstrated trends towards benefit, with major criticisms that recruitment has occurred too late when the hematoma growth has already slowed. Therefore, trials need to be conducted within the very early timeframe, but patients are largely outside this window if they arrive in hospital through standard ambulance transport. As such, prehospital recruitment using a MSU is critical due to the substantial time savings provided for diagnosis after symptom onset. This also means that if future trials do show a significant benefit of hemostatic therapies in hyperacute intracerebral hemorrhage, MSUs will be central to providing treatment within the early time windows.

\section{FUTURE DIRECTIONS}

Although we have shown that a MSU has been successfully operationalized into the ambulance and stroke services in Melbourne, there are research directions and challenges still to be addressed for future operation.

\section{CT scanner limitations}

The availability of cerebral imaging is the unique feature of MSUs that enables pre-hospital stroke treatment. The current CereTom CT scanner has been the standard hardware in almost all first-generation MSUs due to its portability, significantly lower weight, self-mobility, and ability to run from battery power. The trade-off for these features is that there are limitations in image quality, scanner capabilities, and adequate coverage of the head and neck ${ }^{[35]}$.

The overall experience in operating the CereTom scanner in the Melbourne MSU has been that whilst standard non-contrast CT brain sequences are adequate to exclude contraindications to thrombolysis, the substantially slower acquisition time compared to in-hospital scanners allow greater patient movement and resultant artefact. This makes interpretation difficult and may necessitate repeat imaging in some cases, although this rarely affected the ability to administer thrombolysis on the MSU.

The smaller bore hole where the head of the patient rests inside the scanner also presents unique challenges. First, this does not permit imaging of most of the neck and thorax as they cannot fit in the scanner, hence CT-angiogram covers only the intracranial vessels and not usually the common carotid bifurcation or thoracic vessels. This missing information is useful to diagnose underlying stenosis or occlusion of extracranial vessels and, in cases of large vessel occlusion, may help neuro-interventional services plan the procedural approach for EVT. Second, the required head positioning for the small bore hole excludes patients with significant upper spinal kyphosis from being scanned as they are unable to maneuvered into the scanning position. In a similar fashion, the scanner is unable to fully scan the lower posterior fossa in patients placed in a cervical collar or with short necks, as these require the shoulders to enter the bore hole to fully image the brain. Thrombolysis cannot be given with incomplete images; therefore such patients may be unable to benefit from MSU management.

Another limitation is the quality of some CT-angiogram images. The fast acquisition time of in-hospital scanners allow tracking of a test contrast bolus to determine optimal contrast delivery individualized for the patient. Whilst bolus tracking is possible on the CereTom scanner, the slow acquisition time makes this impractical in routine MSU service. As a result, the Melbourne MSU uses set contrast delay timings that are likely to be appropriate for the majority of patients but results in suboptimal CT-angiogram images in 
some cases. This may lead to difficulty in diagnosing large vessel occlusion and need for repeat in-hospital imaging, which will delay commencement of EVT.

The final limitation is the lack of whole-brain CT-perfusion capability. Due to the low number of slices that the CereTom scanner can perform on a $1 \mathrm{~cm}$ thick CT-perfusion slab, the end imaging result is adjudged not sufficient for routine clinical use. The consequences are that firstly, the MSU is unable to provide thrombolysis for suitable patients $>4.5 \mathrm{~h}$ from onset ${ }^{[36]}$ and determining potential EVT-eligible patients $>6 \mathrm{~h}^{[18,19]}$. Secondly, CT-perfusion adds additional diagnostic information where there is uncertainty about a potential non-stroke presentation and helps to decrease thrombolysis of stroke mimics ${ }^{[37]}$. Lastly, the additional information on estimated ischemic penumbra and ischemic core also help refine reperfusion decisions, especially if the patient has risk factors for hemorrhagic transformation ${ }^{[38]}$.

Therefore, there is a need for next generation CT scanning devices that not only deliver better imaging quality and capabilities to better approximate in-hospital scanners, but also maintain the portability that allows fixing into a MSU without needing a chassis that would make operation in crowded cities impractical.

\section{Telemedicine-based operations}

Many of the US-based MSUs utilize telemedicine-based services where the medical practitioner can view an audio-visual feed of the patient and receive transmitted CT images remotely. This has practical advantages in allowing the medical practitioner to conduct other work in between telemedicine consultations and provides potential for one stroke doctor to manage multiple MSUs. However, potential downsides include slower management decisions, as the medical practitioner must rely on other personnel to gather relevant information, as well as potential delays in transmission of imaging. In the worst-case scenario of technical failure, the medical practitioner may be entirely unable to view the patient or not be able to receive imaging. With no alternatives on-board the vehicle, this would force the patient to go to hospital for definitive management, which would be delayed by the aborted MSU review.

The largest telemedicine validation study published by the Houston MSU group found a statistically significant difference in time to thrombolysis decision making between the onboard and telemedicine neurologist, although the absolute median time difference was $3 \min (18 \min v s .21 \min , P=0.01)^{[39]}$. Of note, no telemedicine connection or imaging transmission failures were reported ${ }^{[39]}$. In contrast, telemedicine connectivity was trialed within the Berlin MSU metropolitan dispatch zone with similar telemedicine hardware to the Melbourne MSU. This found that connectivity with $4 \mathrm{G}$ mobile signal failed in $17 \%$ of attempts and $3 \mathrm{G}$ signal in $15 \%$ of attempts ${ }^{[40]}$. With exclusive $4 \mathrm{G}$ signal use on the Melbourne MSU, this error rate would likely be unacceptable for ongoing operation should this be replicated locally.

Further considerations for telemedicine are that much of the initial assessment and decision making for scanning occurs outside the vehicle, whereas the Melbourne MSU audio-visual equipment are fixed inside the back cabin. Waiting for extrication and loading into the vehicle to allow telemedicine connectivity may unduly lengthen the time that the MSU spends at each patient, making the workflow inefficient for cases that are unlikely to be treatment eligible. Portable telemedicine equipment (such as electronic glasses or body cameras) that can be brought away from the vehicle are preferred in this setting but maintaining mobile signal connectivity for a good quality video signal may be challenging. Future implementation of a telemedicine system therefore relies greatly upon technological solutions that offer very high reliability, rapid imaging transmission, and immediate troubleshooting. 


\section{Dispatch specificity}

Dispatch accuracy is a current issue for Melbourne MSU operation. In our region, the currently used emergency dispatch algorithm only has a $32 \%$ concordance with a paramedic diagnosis of stroke or TIA. Not surprisingly, the Melbourne MSU is cancelled by either the first responding paramedic crew or by the MSU personnel in $60 \%$ of cases, mainly due to low likelihood of stroke. This is normally while the vehicle is en route to a case or, in some instances, after the MSU has physically arrived at the address.

This low specificity for stroke is particularly problematic for a single resource like the MSU, as the service cannot attend concurrent dispatches or may be much further away from subsequent dispatched cases. The Melbourne MSU is only attached to $65 \%$ of all potential dispatches in the 20-kilometre dispatch zone and therefore cannot attend all stroke cases within the central operating area. Poor dispatch specificity worsens this situation further if the vehicle is continually dispatched to low likelihood cases, further limiting the availability of the MSU to attend patients who are eligible to receive pre-hospital stroke treatments.

The Berlin MSU group developed a new local dispatch algorithm derived from the most specific stroke symptoms after retrospective analysis of emergency calls ${ }^{[4]}$. This was prospectively evaluated for 274 patients in a 2-month trial after an $1 \mathrm{~h}$ training session with a small group of dispatchers, with the finding of a positive predictive value of $47.8 \%$ for eventual diagnosis of stroke ${ }^{[41]}$. Whilst initially promising, no further validation has been conducted with a broader group of dispatcher personnel over a longer timeframe. In the local Australian setting, there are also practical issues in whether the emergency dispatch organizations are willing to use a bespoke algorithm for a single condition.

Future research in this area must address stroke dispatch in a whole systems approach, both for MSU and regular ambulance dispatch. A multi-tiered approach is required, including consensus for changes to internationally used emergency algorithms, review of local dispatch practices, training of emergency call takers and development of dispatch screening procedures used by each MSU service. This optimization is critical to allow best utilization of the limited number of MSUs worldwide and ensure these high-resource services are cost-effective.

\section{Functional outcomes}

MSUs around the world have consistently demonstrated substantial time savings in commencement of thrombolysis, with evidence for EVT time saving also shown by the Melbourne service. In almost all instances this has led to clear benefits in functional outcomes in published literature ${ }^{[23,26]}$. Two major clinical trials to determine the clinical efficacy of MSUs, based in the US and Germany (Clinicaltrials.gov registrations: BEST-MSU trial NCT02190500 and B_PROUD trial NCT02869386) have recently completed and full results are pending. These utilize pseudo-randomized methods of alternating MSU availability or alternating dispatch regions. These results will be pivotal in future acceptance of MSUs as a gold standard for clinical care, but the translatability of such results to the operational situation in Melbourne needs to be carefully analyzed. Given that the Melbourne MSU shows greater time savings than some international services $^{[2]}$ and is able to provide EVT triage that is not possible in other regions, the potential benefit of the Melbourne service may be greater than benefits reported in the trials.

Outside of clinical trials, the Berlin MSU group has conducted an interim observational analysis of the effect of MSU operation on functional outcomes compared to in-hospital controls ${ }^{[10]}$. When comparing 305 MSU thrombolysis cases to 353 control cases, there was a median time saving of $30 \mathrm{~min}$ (48 $\mathrm{min} v \mathrm{~s}$. $76 \mathrm{~min}, P<$ 0.0005) for time from ambulance dispatch to commencement of thrombolysis in favor of MSU treatment ${ }^{[10]}$. Overall functional outcomes showed that there was a trend towards but no statistically significant benefit on the prespecified primary endpoint of excellent outcome (modified Rankin Scale 0-1, 53\% vs. $47 \%, P=0.14)^{[10]}$. 
However, there were a number of statistically significant secondary outcomes including increased return to ambulation (modified Rankin Scale $0-3,83 \%$ vs. $74 \%, P=0.004)$ and reduced mortality $(6 \% v s .10 \%, P=0.22)^{[10]}$. This study is likely to be underpowered and will need confirmation by upcoming trials. As previously mentioned, the Berlin MSUs do not have the opportunity to provide EVT bypass and triage, therefore the results reflect earlier thrombolysis only.

\section{Health economic analysis}

Preliminary health economic analyses of the Melbourne MSU have been carried out for the first full calendar year of operation ${ }^{[42]}$. The overall cost of running the MSU service was calculated by adding expenditure from setup, wages, maintenance, and depreciation offset by savings from improved triage and streamlining of in-hospital workflow. This was then divided by the disability-adjusted life years avoided by earlier reperfusion therapy on the MSU. The overall cost to save one disability-adjusted life year was estimated in this manner to be between $\$ 19,003-\$ 44,255$ (Australian dollars) in $95 \%$ of model iterations ${ }^{[42]}$, which was below the general $\$ 50,000$ threshold for approval of medicines to the Australian Government funded Pharmaceuticals Benefits Scheme ${ }^{[43]}$. These assumptions, however, still rely on the accuracy of translating time savings to clinical benefit as well as the assumed linearity of this translation, which may not necessarily be the case with thrombolysis where benefit decays faster with time delays during the early time windows ${ }^{[26]}$.

Similar analyses of potential cost-effectiveness have been conducted by the Berlin MSU service, which showed an incremental cost-effectiveness ratio of $€ 32,456$ per quality-adjusted life year ${ }^{[44]}$. This study also relied on estimating improved functional outcome from time savings and utilized running costs unique to this MSU service. In contrast, cost modelling work carried out with the Cleveland MSU showed that, independent of improved outcomes, using a MSU to transport most stroke patients could potentially be cost neutral by avoiding secondary transfers and bypassing emergency department attendance ${ }^{[45]}$. Assumptions here, however, are firmly grounded in a US-based system with very different costs and mix of private funding, which may have limited translatability to other countries.

Overall, initial estimates of the cost-effectiveness of MSUs appear to be comparable with many interventions outside of stroke. Of note, these analyses largely comprise the effect of reperfusion therapy for ischemic stroke without consideration of benefits in other areas, such as intracranial hemorrhage. No doubt the upcoming randomized control trials will provide confirmatory evidence of clinical benefits and allow a wider analysis of cost-effectiveness.

\section{CONCLUSION}

The Melbourne MSU service provides an exciting frontier of pre-hospital stroke care management. The service has been successfully integrated into local Ambulance Victoria operations and has delivered substantial benefits for reperfusion therapy in ischemic stroke and management of intracranial hemorrhage. Future research directions involve evolution of imaging and technical capabilities of MSU services in addition to long term health and cost benefits.

\section{DECLARATIONS}

\section{Authors' contributions}

Substantial contribution to conception, design of the project, and performed data analysis and interpretation: Zhao H, Campbell BCV, Foster S, Stephenson M, Coote S, Langenberg F, Easton D, Donnan GA, Davis SM 


\section{Availability of data and materials}

Data that supports the findings of this research are available upon reasonable request by a suitably qualified investigator.

\section{Financial support and sponsorship}

The Melbourne Mobile Stroke Unit and associated projects received funding from the Australian Commonwealth Government, Victorian State Government, Royal Melbourne Hospital Neurosciences Foundation, Stroke Foundation, The Florey Institute of Neurosciences and Mental Health, University of Melbourne, Boehringer Ingelheim, and private donation.

\section{Conflicts of interest}

Dr. Zhao discloses grants from the Australian Commonwealth Government (MRFF1194787) and the University of Melbourne and personal fees from Boehringer Ingelheim. Prof. Davis discloses grants from the National Health and Medical Research Council and personal fees from Abbott, Boehringer Ingelheim, and Medtronic.

\section{Ethical approval and consent to participate}

Data collection for the Melbourne Mobile Stroke Unit is approved by the Melbourne Health Human Research Ethics Committee with waiver of patient consent (QA2017169).

\section{Consent for publication}

Not applicable.

\section{Copyright}

(C) The Author(s) 2021 .

\section{REFERENCES}

1. Audebert H, Fassbender K, Hussain MS, et al; PRESTO Group. The PRE-hospital Stroke Treatment Organization. Int J Stroke 2017; 12:932-40

2. Zhao H, Coote S, Easton D, et al. Melbourne mobile stroke unit and reperfusion therapy: greater clinical impact of thrombectomy than thrombolysis. Stroke 2020;51:922-30.

3. Walter S, Kostpopoulos P, Haass A, et al. Bringing the hospital to the patient: first treatment of stroke patients at the emergency site. PLoS One 2010;5:e13758.

4. Ebinger M, Winter B, Wendt M, et al. Effect of the use of ambulance-based thrombolysis on time to thrombolysis in acute ischemic stroke: a randomized clinical trial. JAMA 2014;311:1622-31.

5. Parker SA, Bowry R, Wu TC, et al. Establishing the first mobile stroke unit in the United States. Stroke 2015;46:1384-91.

6. Zaidat OO, Changal KH, Sultan-Qurraie A, et al. Diurnal variations in the first 24/7 mobile stroke unit. Stroke 2019;50:1911-4.

7. Parker SA, Kus T, Bowry R, et al. Enhanced dispatch and rendezvous doubles the catchment area and number of patients treated on a mobile stroke unit. J Stroke Cerebrovasc Dis 2020;29:104894.

8. Zhao H, Pesavento L, Coote S, et al. Ambulance clinical triage for acute stroke treatment: paramedic triage algorithm for large vessel occlusion. Stroke 2018;49:945-51.

9. Ebinger M, Kunz A, Wendt M, et al. Effects of golden hour thrombolysis: a prehospital acute neurological treatment and optimization of medical care in stroke (PHANTOM-S) substudy. JAMA Neurol 2015;72:25-30.

10. Kunz A, Ebinger M, Geisler F, et al. Functional outcomes of pre-hospital thrombolysis in a mobile stroke treatment unit compared with conventional care: an observational registry study. Lancet Neurol 2016;15:1035-43.

11. Audebert H, Ebinger M, Siegerink B, et al. The effects of Mobile Stroke Units on functional outcome after cerebral ischemia: B_PROUD. International Stroke Conference 2020. Los Angeles, USA.

12. Bowry R, Parker S, Rajan SS, et al. Benefits of stroke treatment using a mobile stroke unit compared with standard management: the BEST-MSU study run-in phase. Stroke 2015;46:3370-4.

13. Shownkeen H., Mohajer-Esfahani M., Crumlett H., Lindstrom A. E-112 a single center comparison of stroke treatment times: impact of a mobile stroke unit. J Neurointerv Surg 2018;10:A103-4.

14. Lin E., Calderon V., Goins-Whitmore J., Bansal V., Zaidat O. World's first 24/7 mobile stroke unit: initial 6-month experience at Mercy Health in Toledo, Ohio. Front Neurol 2018;9:283. 
15. Kim JT, Fonarow GC, Smith EE, et al. Treatment with tissue plasminogen activator in the golden hour and the shape of the 4.5-hour timebenefit curve in the National United States Get with the guidelines-stroke population. Circulation 2017;135:128-39.

16. Zhao $\mathrm{H}$, Coote $\mathrm{S}$, Pesavento $\mathrm{L}$, et al. Prehospital idarucizumab prior to intravenous thrombolysis in a mobile stroke unit. Int $J$ Stroke 2019;14:265-9.

17. Fassbender K, Grotta JC, Walter S, Grunwald IQ, Ragoschke-Schumm A, Ragoschke-Schumm A. Mobile stroke units for prehospital thrombolysis, triage, and beyond: benefits and challenges. Lancet Neurol 2017;16:227-37.

18. Nogueira RG, Jadhav AP, Haussen DC, et al; DAWN Trial Investigators. Thrombectomy 6 to 24 hours after stroke with a mismatch between deficit and infarct. $N$ Engl J Med 2018;378:11-21.

19. Albers GW, Marks MP, Kemp S, et al; DEFUSE 3 Investigators. Thrombectomy for stroke at 6 to 16 hours with selection by perfusion imaging. N Engl J Med 2018;378:708-18.

20. Czap AL, Grotta JC, Parker SA, et al. Emergency department door-to-puncture time since 2014. Stroke 2019;50:1774-80.

21. Patel P, Sheikhi L, Mullaguri N, et al. Abstract WP226: bypassing interhospital transfers for large vessel occlusions in the era of mobile stroke units. Stroke 2018;49.

22. Kerrigan D, Di Lorenzo R, Nour M, Russman A, Uchino K, Hussain MS. Abstract TMP19: mobile stroke units have higher rates of recanalization of emergent large vessel occlusion. Stroke 2019;50.

23. Saver JL, Goyal M, van der Lugt A, et al; HERMES Collaborators. Time to treatment with endovascular thrombectomy and outcomes from ischemic stroke: a meta-analysis. JAMA 2016;316:1279-88.

24. Meretoja A, Keshtkaran M, Saver JL, et al. Stroke thrombolysis: save a minute, save a day. Stroke 2014;45:1053-8.

25. Meretoja A, Keshtkaran M, Tatlisumak T, Donnan GA, Churilov L. Endovascular therapy for ischemic stroke: save a minute-save a week. Neurology 2017;88:2123-7.

26. Emberson J, Lees KR, Lyden P, et al. Effect of treatment delay, age, and stroke severity on the effects of intravenous thrombolysis with alteplase for acute ischaemic stroke: a meta-analysis of individual patient data from randomised trials. Lancet 2014;384:1929-35.

27. Al-shahi Salman R, Frantzias J, Lee RJ, et al. Absolute risk and predictors of the growth of acute spontaneous intracerebral haemorrhage: a systematic review and meta-analysis of individual patient data. Lancet Neurol 2018;17:885-94.

28. Sprigg N, Flaherty K, Appleton JP, et al. Tranexamic acid for hyperacute primary intracerebral haemorrhage (TICH-2): an international randomised, placebo-controlled, phase 3 superiority trial. Lancet 2018;391:2107-21.

29. Gladstone DJ, Aviv RI, Demchuk AM, et al. Effect of recombinant activated coagulation factor VII on hemorrhage expansion among patients with spot sign-positive acute intracerebral hemorrhage: the SPOTLIGHT and STOP-IT randomized clinical trials. JAMA Neurol 2019;76:1493-501.

30. Mayer SA, Brun NC, Begtrup K, et al. Efficacy and safety of recombinant activated factor VII for acute intracerebral hemorrhage. $N$ Engl J Med 2008;358:2127-37.

31. Li Q, Warren AD, Qureshi AI, et al. Ultra-early blood pressure reduction attenuates hematoma growth and improves outcome in intracerebral hemorrhage. Ann Neurol 2020;88:388-95.

32. Connolly SJ, Crowther M, Eikelboom JW, et al. Full study report of andexanet alfa for bleeding associated with factor Xa inhibitors. $N$ Engl J Med 2019;380:1326-35.

33. Huttner HB, Schellinger PD, Hartmann M, et al. Hematoma growth and outcome in treated neurocritical care patients with intracerebral hemorrhage related to oral anticoagulant therapy: comparison of acute treatment strategies using vitamin $\mathrm{K}$, fresh frozen plasma, and prothrombin complex concentrates. Stroke 2006;37:1465-70.

34. Abid KA, Vail A, Patel HC, King AT, Tyrrell PJ, Parry-Jones AR. Which factors influence decisions to transfer and treat patients with acute intracerebral haemorrhage and which are associated with prognosis? A retrospective cohort study. BMJ Open 2013;3:e003684.

35. John S, Stock S, Cerejo R, et al. Brain imaging using mobile CT: current status and future prospects. J Neuroimaging 2016;26:5-15.

36. Ma H, Campbell BCV, Parsons MW, et al. Thrombolysis guided by perfusion imaging up to 9 hours after onset of stroke. $N$ Engl J Med 2019;380:1795-803.

37. Campbell BC, Weir L, Desmond PM, et al. CT perfusion improves diagnostic accuracy and confidence in acute ischaemic stroke. $J$ Neurol Neurosurg Psychiatry 2013;84:613-8.

38. Campbell BCV, Khatri P. Stroke. Lancet 2020;396:129-42.

39. Bowry R, Parker SA, Yamal JM, et al. Time to decision and treatment with tPA (tissue-type plasminogen activator) using telemedicine versus an onboard neurologist on a mobile stroke unit. Stroke 2018;49:1528-30.

40. Winter B, Wendt M, Waldschmidt C, et al. $4 \mathrm{G}$ versus $3 \mathrm{G}$-enabled telemedicine in prehospital acute stroke care. Int $J$ Stroke 2019;14:620-9.

41. Krebes S, Ebinger M, Baumann AM, et al. Development and validation of a dispatcher identification algorithm for stroke emergencies. Stroke 2012;43:776-81.

42. Kim J, Easton D, Zhao H, et al. Economic evaluation of the Melbourne mobile stroke unit. Int J Stroke 2020;1747493020929944.

43. Wang S., Gum D., Merlin T. Comparing the ICERs in medicine reimbursement submissions to NICE and PBAC-Does the presence of an explicit threshold affect the ICER proposed? Value Health 2018;21:938-43.

44. Gyrd-Hansen D, Olsen KR, Bollweg K, Kronborg C, Ebinger M, Audebert HJ. Cost-effectiveness estimate of prehospital thrombolysis: results of the PHANTOM-S study. Neurology 2015;84:1090-7.

45. Reimer AP, Zafar A, Hustey FM, et al. Cost-consequence analysis of mobile stroke units vs. standard prehospital care and transport. Front Neurol 2019;10:1422. 